www.jmscr.igmpublication.org

Impact Factor 5.84

Index Copernicus Value: 71.58

ISSN (e)-2347-176x ISSN (p) 2455-0450

crossref DOI: _https://dx.doi.org/10.18535/jmscr/v5i9.74

\title{
Effect of Drotavarine Hydrochloride and A Combination of - Hyoscine-N- Butyl Bromide With Valethamate Bromide on the Duration of Active Phase of Labor - A Comparative Study
}

\author{
Authors \\ Surbhi Saini, Balwinder Kaur, Arvinder Kaur \\ Department of Obstetrics and Gynaecology, Govt. Medical College, Patiala, Punjab, India \\ Corresponding Author \\ Balwinder Kaur \\ Associate Professor \\ 360 North Avenue, Bhadson Road Patiala Punjab India, 147001 \\ Email: drkaurbalwinder@gmail.com,+919814486292
}

\begin{abstract}
Labor, an important event, is marked by the fear of pain. Painless and short labor is desired. Obstetricians goal is, healthy neonate and mother. Methods used to shorten active phase of labor are welcomed. Prolonged labor causes high feto-maternal morbidity. Shortening of duration of labor, without jeopardizing feto- maternal interests is warranted; Various methods tried to accelerate labor. Hyoscine-n-butyl bromide, scopolamine and Valethamate bromide widely studied. Present study conducted to compare effects of Drotavarine hydrochloride and combination of Hyoscine-N-butyl bromide plus Valethamate bromide on duration of active phase and their feto-materal side effects. 200 women divided into two groups 100 each. Group A received Drotavarine hydrochloride 40mg I/M, 2 doses 2 hours apart( $2^{\text {nd }}$ if required). Group B given combination of Hyoscine-N-butyl bromide $40 \mathrm{mg}$ and Valethamate bromide 16mg I/M, 2 doses 2 hours apart(2nd if required). 98\% delivered vaginally. $2 \%$ (LSCS) in each group and excluded. Mean no. of doses $1.36 \pm 0.48$ (Group A), $1.83 \pm 0.37$ (Group B) p value $<0.001$ HS. Mean rate of dilatation $(\mathrm{cms} / \mathrm{hr})$ was more in Group A(3.81 \pm 1.52$)$ than $B(2.29 \pm 0.64)$ p value $<0.001$ HS. Mean Active phase was $112.70 \pm 53.09$ mins in Group $A$ and $171.44 \pm 57.24 \mathrm{~min}$ in $B, p$ value $<0.001$ HS. Drug-delivery interval was $45.10 \pm 63.22 \mathrm{~min}$ (groupA) and $202.09 \pm 60.35 \mathrm{~min}$ (Group B) p value $<0.001 \mathrm{HS}$. No serious feto-maternal side effects. Drotavarine and combination drugs are safe to shorten the duration of active phase.

Keywords: Drotavarine, Valethamate, active phase. Dilatation.
\end{abstract}

\section{Introduction}

Labor, an important event in woman's life, is marked by fear of pain. Painless and short labor is a constant aim for obstetricians. ${ }^{[1]} \mathrm{Goal}$ is, healthy mother and neonate, avoiding cesarean and its complications. Methods that aim at minimizing, functional cervical dystocia and cutting short active phase of first stage of labor are welcomed..
With initiation of parturition, the cervix must soften, yield and become more readily dilatable.

Duration of Labor

\begin{tabular}{|l|c|c|}
\hline Stages & Primigravida & Multigravida \\
\hline First & & \\
Latent Phase & $6-8 \mathrm{hr}$ & $3-5 \mathrm{hr}$ \\
Active Phase & $3-5 \mathrm{hr}$ & $1-3 \mathrm{hr}$ \\
\hline Second & $1-1.5 \mathrm{hr}$ & $0.5 \mathrm{hr}$ \\
\hline Third & $15 \mathrm{mins}$ & $15 \mathrm{mins}$ \\
\hline
\end{tabular}


Prolonged Labor: General consensus, labor lasting > 24 hours is prolonged labor.

Active phase is arbitrarily defined prolonged, when its duration exceeds 18 hours in primigravida, 12 hours in multi gravida. Definition, generally denotes prolongation of the first stage of labor associated with slow dilatation of cervix. ${ }^{[2]}$ Prolonged labor causes high feto-maternal morbidity, thus shortening of duration of labor, without jeopardizing fetal and maternal interests is warranted. ${ }^{[3]}$ Cervical dilatation, an important factor, determines duration of labor and is result of the driving force of uterine contractions acting against passive tissue resistance. ${ }^{[4]}$ Pattern of cervical dilatation, during normal labor is sigmoid shaped (Friedman's Partograph ${ }^{[5]}$ )

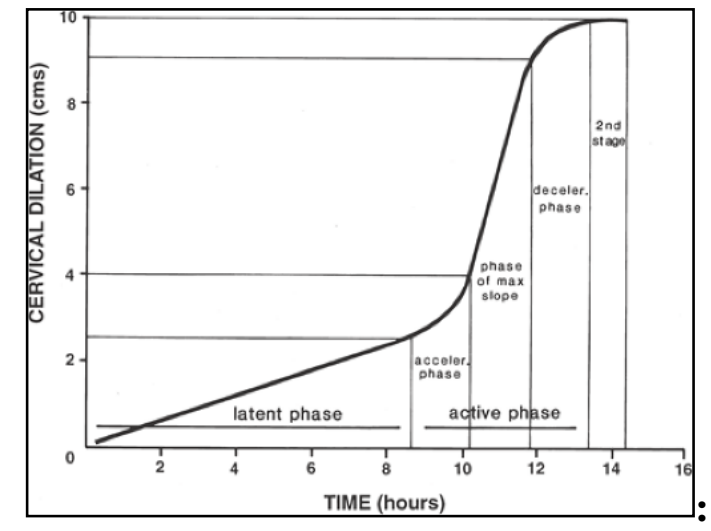

Friedman's Partograph

Later, Alert and action lines introduced ${ }^{[6]}$ WHO model of partograph ${ }^{[7,8]}$ devised.

Simplified WHO Partograph, is commonly used to assess progress of labor.

Methods to accelerate process and facilitate cervical dilatation, are:

- Sweeping membranes and stretching of cervix releases local prostaglandin E2 from chorio-amniotic membranes, decidua, cervix. $^{[9]}$

- Amniotomy ${ }^{[10,11]}$, prostaglandins synthesis is increased which can induce, modulate labor and ripen the cervix. ${ }^{[9]}$

- Prostaglandins for induction of labor especially $\mathrm{PGE}_{1}$ and $\mathrm{PGE}_{2}$ gel for cervical ripening.
- Oxytocin ${ }^{[12]}$ Commonly used for induction and augmentation of labor. Amniotomy can be combined with oxytocin.

- Hyoscine-n-butyl bromide and scopolamine - used for pain relief and cervical dilatation resulting in shortening of labor.

- Valethamate bromide resulls in relaxation of cervical musculature leading to quick dilatation and shortening labor..

- Drotavarine hydrochloride- acts as antispasmodic in addition to causing cervical dilatation..

\section{Aims and Objectives}

1. To compare effects of Drotavarine hydrochloride and combination of Hyoscine-N-butyl bromide with Valethamate bromide on duration of active phase of labor.

2. To evaluate incidence of Feto-Maternal side effects.

\section{Material and Methods}

200 women admitted in Labor room of Department of Obstetrics and Gynaecology, G.M.C. Patiala were enrolled.

\section{Inclusion criteria}

1. Singleton term pregnancy

2. Cephalic presentation

3. Uterine contractions $\geq 2 / 10$ minutes, each lasting for at least 20 seconds

4. Cervical dilatation of $\geq 4 \mathrm{~cm}$

5. Willingness to participate.

6.

\section{Exclusion criteria}

1. Malpresentation

2. Twins

3. Previous Cervical surgery / injury.

4. Medical disorders.

5. Hypersensitivity to drugs

6. Other spasmolytic, if used within 48 hours.

7. Fetal / maternal distress

8. Absolute indication for cesarean section

History esp. period of gestation, duration of onset of labor pains, bleeding or leaking $\mathrm{P} / \mathrm{V}$, Obstetric 
history, including outcome of previous pregnancies., past and family history were obtained.

Thorough general and systemic examination was done. Fundal height, fetal lie and presentation,. frequency and intensity of contractions recorded. Fetal monitoring. done by noting rate, rhythm and regularity of fetal heart., Cervical dilatation, effacement,. Bishop's Score and station of presenting part were noted. Pelvic contraction or cephalopelvic disproportion.excluded.

Women, randomly divided into two groups.

\section{Group A}

100 women, received 40mg Drotavarine hydrochloride, I/M, repeated after 2 hours if full dilatation not achieved (maximum 2 doses).

\section{Group B}

100 women, received combination of $40 \mathrm{mg}$ Hyoscine-n-butyl bromide and $16 \mathrm{mg}$ Valethamate bromide, I/M, repeated after 2 hours if full dilatation not achieved (maximum 2 doses).

Drugs were administered in active labor.

Labor monitored. by WHO simplified partograph

- Fetal heart auscultated / half hourly to note rate and rhythm.

- Frequency, intensity and duration of contractions.

- Cervical dilatation by $\mathrm{P} / \mathrm{V}$ examination two hours after first dose and then accordingly.

Low amniotomy done

\section{Progress assessed by}

- P/A examination, noting descent of fetal head

- $\mathrm{P} / \mathrm{V}$ examination for cervical dilatation.

- FHR. monitoring

- Side effects: Maternal tachycardia, dryness of mouth, headache, fever and flushing of face observed.

- Mode and time of delivery noted. Cervix, vagina examined for any trauma.

- Postpartum period observed.

- APGAR at 1, 5 minutes and NICU admission noted.
Drug withdrawn, if fetal / maternal distress and measures to affect delivery were immediately taken.

\section{Parameters studied}

- Duration of active phase, calculated from time of first dose of drug to full dilatation of cervix.

- Rate of cervical dilatation.

- Duration of second stage.

- Duration of third stage.

- Mode of delivery ie vaginal, instrumental or cesarean section.

- Duration of drug-delivery interval calculated from time of first injection till the delivery of baby.

- Third stage complication

- Maternal complications

- Fetal outcome: APGAR score at 1,5 minutes and NICU admission,

Data obtained, recorded, compiled, tabulated and analyzed by Chi square statistics for both groups. Observations and results compared.

\section{Observations and Results}

In both groups, majority women were 21-30 years of age. Mean age was $24.38 \pm 3.123$ years (Group A) , 23.25 \pm 2.88 years(Group B.) Group A, had $45 \%$ primigravida and $55 \%$ multigravida. Group B, $51 \%$ primigravidae and $49 \%$ multigravidae. Mean period of gestation was $38.56 \pm 1.03$ weeks (group A) and 38.69 \pm 0.90 weeks (group B.) Drugs were administered in established labor $(\geq 2$ contractions /10 minutes and cervical dilatation $\geq 4 \mathrm{~cm}$.)

Mean cervical effacement at time of first dose, in both groups was 60-70\%. \{Group A (61.20 \pm 10.37 $\%)$, Group B $(60.20 \pm 10.04 \%)\}$. In each group, $98 \%$ delivered vaginally without any aid. 2\% underwent LSCS in each group. 2 primigravidae (Group A) underwent LSCS due to fetal bradycardia after first dose of Drotavarine. In Group B, 1 primigravida underwent LSCS due to fetal bradycardia and 1 multigravida had uterine inertia despite oxytocin augmentation after 2 


\section{JMSCR Vol||05||Issue||09||Page 27792-27799||September}

doses of drugs. These Women were eliminated from study.

Table 1 Number of Doses Administered

\begin{tabular}{|l|c|c|c|c|}
\hline \multirow{2}{*}{ DOSES } & \multicolumn{2}{|c|}{$\begin{array}{c}\text { GROUP A } \\
\text { (N=98) }\end{array}$} & \multicolumn{2}{c|}{$\begin{array}{c}\text { GROUP B } \\
\text { (N=98) }\end{array}$} \\
\cline { 2 - 5 } & No. & \%age & No. & \%age \\
\hline 1 & 64 & $65.3 \%$ & 17 & $17.3 \%$ \\
\hline 2 & 36 & $36.7 \%$ & 81 & $82.6 \%$ \\
\hline Mean \pm S.D. & \multicolumn{2}{|c|}{$1.36 \pm 0.48$ dose } & $1.83 \pm 0.37$ dose \\
\hline$p$ value & \multicolumn{3}{|c|}{$<0.001$} \\
\hline Significance & \multicolumn{3}{|c|}{ HS } \\
\hline
\end{tabular}

65.3\% women in Group A delivered after single dose and $36.7 \%$ required second dose. In Group B, $17.3 \%$ delivered after single dose and $82.6 \%$ required second. Mean doses was $1.36 \pm 0.48$ (Group A) and 1.83 \pm 0.37 (group B). Group B often needed a second dose, difference statistically significant $(\mathrm{p}<0.001)$.

Table. 2 Rate of Cervical Dilatation

\begin{tabular}{|c|c|c|c|c|}
\hline \multirow{2}{*}{$\begin{array}{l}\text { Rate } \\
(\mathrm{cm} / \mathrm{hr})\end{array}$} & \multicolumn{2}{|c|}{$\begin{array}{c}\text { Group A } \\
(\mathrm{N}=98)\end{array}$} & \multicolumn{2}{|c|}{$\begin{array}{c}\text { Group B } \\
(\mathrm{N}=98)\end{array}$} \\
\hline & No. & \%age & No. & \%age \\
\hline$<1$ & 0 & $0 \%$ & 1 & $1.02 \%$ \\
\hline $1-1.5$ & 6 & $6.12 \%$ & 7 & $7.14 \%$ \\
\hline $1.5-2$ & 8 & $8.16 \%$ & 33 & $33.67 \%$ \\
\hline $2-2.5$ & 4 & $4.08 \%$ & 26 & $26.53 \%$ \\
\hline $2.5-3$ & 22 & $22.44 \%$ & 23 & $23.46 \%$ \\
\hline $3-3.5$ & 6 & $6.12 \%$ & 5 & $5.10 \%$ \\
\hline 3.5- 4 & 21 & $21.42 \%$ & 3 & $3.06 \%$ \\
\hline$\geq 4$ & 31 & $31.63 \%$ & 0 & $0 \%$ \\
\hline Mean \pm S.D & \multicolumn{2}{|c|}{$3.81 \pm 1.52$} & \multicolumn{2}{|c|}{$2.29 \pm 0.64$} \\
\hline$p$ value & \multicolumn{4}{|c|}{$<0.001$} \\
\hline Significance & \multicolumn{4}{|c|}{ HS } \\
\hline
\end{tabular}

Rate was $\geq 2 \mathrm{~cm} / \mathrm{hr}$ in $85.69 \%$ (Group A), $58.15 \%$ (Group B).

Mean rate was $3.81 \pm 1.52 \mathrm{~cm} / \mathrm{hr}$ (Group A) and $2.29 \pm 0.64 \mathrm{~cm} / \mathrm{hr}$ (group B). Cervical dilatation was fast with Drotavarine. Difference statistically significant. $(p<0.001)$.

Table 3 Duration of Active Phase

\begin{tabular}{|l|c|c|c|c|}
\hline \multirow{2}{*}{$\begin{array}{l}\text { Duration } \\
\text { (mins) }\end{array}$} & \multicolumn{2}{|c|}{$\begin{array}{c}\text { Group A } \\
\text { (N=98) }\end{array}$} & \multicolumn{2}{c|}{$\begin{array}{c}\text { Group B } \\
\text { (N=98) }\end{array}$} \\
\cline { 2 - 5 } & No. & $\%$ age & No. & $\%$ age \\
\hline$<120$ & 56 & $57.14 \%$ & 8 & $8.16 \%$ \\
\hline $120-240$ & 39 & $39.79 \%$ & 83 & $84.69 \%$ \\
\hline$\geq 240$ & 3 & $3.06 \%$ & 7 & $7.14 \%$ \\
\hline Mean \pm S.D. & $112.70 \pm 53.09$ min & \multicolumn{2}{c|}{$171.44 \pm 57.24$ min } \\
\hline$p$ value & \multicolumn{3}{|c|}{$<0.001$} \\
\hline Significance & \multicolumn{3}{|c|}{ HS } \\
\hline
\end{tabular}

Mean duration of active phase was $112.70 \pm 53.09$ mins (group A), 171.44 \pm 57.24 mins (Group B).

Duration of active phase was shorter in Group A as compared to Group B. Difference statistically highly significant $(p<0.001)$. Mean duration of second and third stages were unaffected in either group.

Table 4 Drug Delivery Interval

\begin{tabular}{|l|c|c|c|c|}
\hline \multirow{2}{*}{$\begin{array}{l}\text { Drug- Delivery } \\
\text { Interval } \\
(\mathrm{min})\end{array}$} & \multicolumn{2}{|c|}{$\begin{array}{c}\text { GROUP A } \\
(\mathrm{N}=98)\end{array}$} & \multicolumn{2}{c|}{$\begin{array}{c}\text { GROUP B } \\
(\mathrm{N}=98)\end{array}$} \\
\cline { 2 - 5 } & No. & $\%$ age & No. & $\%$ age \\
\hline$<60$ & 1 & $1.02 \%$ & 0 & $0 \%$ \\
\hline $60-90$ & 16 & $16.32 \%$ & 0 & $0 \%$ \\
\hline $90-120$ & 29 & $29.59 \%$ & 3 & $3.06 \%$ \\
\hline $120-150$ & 22 & $22.44 \%$ & 13 & $13.26 \%$ \\
\hline$\geq 150$ & 32 & $32.65 \%$ & 82 & $83.67 \%$ \\
\hline Mean \pm S.D. & $145.10 \pm 63.22 \mathrm{~min}$ & $202.09 \pm 60.35 \mathrm{~min}$ \\
\hline$p$ value & \multicolumn{6}{|c|}{$<0.001$} \\
\hline Significance & \multicolumn{3}{|c|}{ HS } \\
\hline
\end{tabular}

$55.91 \%$ delivered within 120 mins ( Group A) and $3.06 \%$ ( Group B ) where $83.67 \%$ took $>150$ mins to deliver compared to $32.65 \%$ ( Group A). Mean drug-delivery interval was $145.10 \pm 63.22$ mins ((Group A) and 202.09 \pm 60.35 mins (Group B.) Result statistically significant $(p<0.001)$.

Third stage Complications: 1 case of retained placenta in Group A which was partially adherent.

Table 5 Maternal Side Effects

\begin{tabular}{|l|c|c|c|c|}
\hline \multirow{2}{*}{ Side Effects } & \multicolumn{2}{|c|}{$\begin{array}{c}\text { GROUP A } \\
\text { (N=98) }\end{array}$} & \multicolumn{2}{c|}{$\begin{array}{c}\text { GROUP B } \\
\text { (N=98) }\end{array}$} \\
\cline { 2 - 5 } & No. & $\%$ age & No. & $\%$ age \\
\hline Tachycardia & 5 & $5.10 \%$ & 8 & $8.16 \%$ \\
\hline Dryness of Mouth & 6 & $6.12 \%$ & 3 & $3.06 \%$ \\
\hline Headache & 4 & $4.08 \%$ & 1 & $1.02 \%$ \\
\hline Flushing of Face & 0 & $0 \%$ & 0 & $0 \%$ \\
\hline Rise In Temperature & 0 & $0 \%$ & 0 & $0 \%$ \\
\hline$p$ value & \multicolumn{4}{|c|}{0.202} \\
\hline Significance & \multicolumn{4}{|c|}{ NS } \\
\hline
\end{tabular}

Group A: Dryness of mouth $(6.12 \%$,), headache (4.08\%), tachycardia (5.10\%) compared to Group B with dryness of mouth (3.06\%), headache (1.02\%), tachycardia(8.16\%) which subsided after maternal hydration. Flushing of face or fever not reported in either group. Side effects were minor, did not continue in postpartum period, subsided without any specific treatment and statistically not significant. 
Neonatal well being, evaluated by Virginia APGAR at 1, 5 minutes.. Majority had APGAR scores 9,9 in both groups (mean APGAR $8.96 \pm 0.281$ and $9.0 \pm 0.00$ at 1 and 5 mins respectively).

In Group B, 1 NICU admission, because of low birth weight. No neonatal morbidity in either group within 48 hours. APGAR score was not affected and difference not statistically significant..

\section{Discussion}

Prolonged labor has implications for mother and fetus. Reducing duration of labor is highly desirable goal, for maternal and fetal well being. [8] 'Active management of labor 'was conceptualized by O'Driscoll in 1973. It helped in reducing maternal and perinatal morbidity and mortality. Cervical dilatation, is important factor which determines duration of labor. Relieving pain and shortening duration of active phase will have beneficial effect on mother and fetus. Various drugs tried to hasten cervical dilatation and minimize pain without increasing maternal or perinatal mortality and morbidity. Drotavarine hydrochloride, Valethamate bromide and Hyoscine-N-butyl bromide have been studied in the past..

Table 6 Mean Rate of Cervical Dilatation

\begin{tabular}{|l|c|c|}
\hline STUDY & $\begin{array}{c}\text { GROUP A } \\
(\mathrm{cm} / \mathrm{hr})\end{array}$ & $\begin{array}{c}\text { GROUP B } \\
(\mathrm{cm} / \mathrm{hr})\end{array}$ \\
\hline Sharma et al $(2001)^{[10]}$ & 2.04 & 1.84 \\
\hline Madhu et al (2010) ${ }^{[12]}$ & $3 \pm 1.4$ & $2.4 \pm 0.9$ \\
\hline${\text { Nagaria et al (2009) }{ }^{[1]}}^{\text {Naga et al }(2014)^{[13]}}$ & 3.38 & 2.17 \\
\hline Present study (2016) & 1.97 & 1.45 \\
\hline
\end{tabular}

Mean rate in our study was $3.81 \pm 1.52 \mathrm{~cm} / \mathrm{hr}$ (Group A), $2.29 \pm 0.64 \mathrm{~cm} / \mathrm{hr}$ (GroupB). Drotavarine has tened cervical dilatation more than other drug as seen in studies conducted by Sharma et $\mathrm{al}^{[10]}$, Nagaria et $\mathrm{al}^{[11]}$, Madhu et al ${ }^{[12]}$, Naga et $\mathrm{al}^{[13]}$. Result with I/M Drotavarine (present study) was better than I/V Drotavarine (Naga et al. ${ }^{[13]}$ ). 2 doses of Drotavarine (Present study) gave better result than single dose ( Sharma et $\mathrm{al}^{[10]}$ )
Rate of dilatation was more with combination drugs, (our study), than Naga et $\mathrm{al}^{[13]}$ who used $8 \mathrm{mg}$ Valethamate with 40mg Hyoscine-N-butyl bromide half hourly for 3 doses. Combination of drugs (our study) were better than individual drugs (Madhu et $\mathrm{al}^{[12])}$, Sharma et $\mathrm{al}^{[10]}$ and, Nagaria et al. ${ }^{[11]}$

Table. 7 Mean Duration of Active Phase

\begin{tabular}{|l|c|c|}
\hline STUDY & $\begin{array}{c}\text { GROUP A } \\
\text { (minutes) }\end{array}$ & $\begin{array}{c}\text { GROUP B } \\
\text { (minutes) }\end{array}$ \\
\hline Nagaria et al $(2009)^{[11]}$ & $113.51 \pm 74.23$ & $177.40 \pm 105.68$ \\
\hline Kaur et al $(2013)^{[14]}$ & $141.40 \pm 55.41$ & $181.46 \pm 75.58$ \\
\hline Naga et al $(2014)^{[13]}$ & 191 & 256 \\
\hline Edessy et al $(2015)^{[15]}$ & 126 & 138 \\
\hline Patil et al $(2015)^{[16]}$ & $113.2 \pm 60.8$ & $158.78 \pm 58.9$ \\
\hline Present study $(2016)$ & $112.70 \pm 53.09$ & $171.44 \pm 57.24$ \\
\hline
\end{tabular}

Present study: Mean duration was 112.70 \pm 53.09 mins (Group A), 171.44 \pm 57 mins (Group B) and statistically significant. Drotavarine reduced the duration more than other drug (Nagaria et $\mathrm{al}^{[11])}$, Naga et $\mathrm{al}^{[13]}$, Edessy et $\mathrm{al}^{[15]}$ and Patil et $\mathrm{al}^{[16]}$.) I/M Drotavarine (present study) gave better result than I/V (Naga et al. ${ }^{[13]}$ ) as Drotavarine has sustained action. Valethamate group result was comparable to that of Kaur et $\mathrm{al}^{[14]}$ (same combination of drugs but anafortan instead of Drotavarine). Our result was comparable to Nagaria et $\mathrm{al}^{[11]}$ who used Valethamate $16 \mathrm{mg}$ infusion in dextrose. Our result was better than Naga et $\mathrm{al}^{[13]}$ who used $8 \mathrm{mg}$ Valethamate 3 doses/ 1/2 hourly..

Table 8 Doses Required for Successful Delivery

\begin{tabular}{|c|c|c|c|c|c|c|}
\hline \multirow{2}{*}{ STUDY } & \multicolumn{3}{|c|}{$\begin{array}{c}\text { GROUP A } \\
\text { (doses) }\end{array}$} & \multicolumn{3}{|c|}{$\begin{array}{c}\text { GROUP B } \\
\text { (doses) }\end{array}$} \\
\hline & 1 & 2 & 3 & 1 & 2 & 3 \\
\hline Sharma et al (2001) $)^{[10]}$ & $10 \%$ & $66 \%$ & $24 \%$ & - & $6 \%$ & $94 \%$ \\
\hline Patil et al (2015) ${ }^{[16]}$ & $62 \%$ & $30 \%$ & $8 \%$ & $12 \%$ & $30 \%$ & $58 \%$ \\
\hline Selvaraj et al (2016) ${ }^{[18]}$ & - & $96 \%$ & $4 \%$ & - & - & $98 \%$ \\
\hline Present study (2016) & $64 \%$ & $36 \%$ & - & $17 \%$ & $81 \%$ & - \\
\hline
\end{tabular}

$64 \%$ delivered with single dose of Drotavarine whereas $36 \%$ required second. 17\% (group B) delivered after first dose, $81 \%$ after second. Our result is comparable to Patil et $\mathrm{al}^{[16]}$ where majority delivered after single dose of Drotavarine In our study $98 \%$ (Group B) delivered after 2 


\section{JMSCR Vol||05||Issue||09||Page 27792-27799||September}

doses as compared to $42 \%$ ( Patil et al ${ }^{[16]}$ ) where only Valethamate bromide given. Sharma et $\mathrm{al}^{[10]}$ and Selvaraj et $\mathrm{al}^{[18]}$ showed, majority needed second dose. They included primigravidae only. Majority (group B), delivered after a minimum of 3 doses of $8 \mathrm{mg}$ Valethamate bromide (authors ${ }^{[10,16,18])}$. In present study, 16mg Valethamate bromide with 40mg Hyoscine-Nbutyl bromide was given in 2 doses 2 hours apart resulting $81 \%$ vaginal delivery, concluding that this dosage regime is also effective.

Table 9 Drug- Delivery Interval

\begin{tabular}{|l|c|c|}
\hline STUDY & $\begin{array}{c}\text { GROUP A } \\
\text { (minutes) }\end{array}$ & $\begin{array}{c}\text { GROUP B } \\
\text { (minutes) }\end{array}$ \\
\hline $\begin{array}{l}\text { Sharma et al (2001) } \\
{[10]}\end{array}$ & $194 \pm 57.04$ & $220.7 \pm 86.12$ \\
\hline $\begin{array}{l}\text { Nagaria et al } \\
(2009)^{[11]}\end{array}$ & $135.87 \pm 85.94$ & $200.13 \pm 15.04$ \\
\hline Madhu et al (2010) & 183.2 & 206.5 \\
\hline Dahal et al (2013) $)^{[17]}$ & $192.56 \pm 75.47$ & $249.13 \pm 88.32$ \\
\hline $\begin{array}{l}\text { Selvaraj al } \\
(2016)^{[18]} \text { et }\end{array}$ & $219.6 \pm 37.9$ & $274.84 \pm 29.09$ \\
\hline Present study (2016) & $145.10 \pm 63.22$ & $202.09 \pm 60.35$ \\
\hline
\end{tabular}

Our study; Drug- delivery interval was 145.10 \pm 63.22 mins (Group A) 202.09 \pm 60.35 mins (Group B).Drotavarine hastens process compared to other drug (authors. ${ }^{[10,11,12,17,18])}$ Drotavarine 2 doses (Our study) gave better result than single dose (Sharma et al. ${ }^{[10])}$

Selvaraj et $\mathrm{al}^{[18]}$, Sharma et al ${ }^{[10]}$ showed longer drug delivery interval as they studied primigravidae alone. Drug delivery interval was slightly better with combinanation (present study) than individual drugs( Selvaraj et al ${ }^{[18]}$, Madhu et al ${ }^{[12]}$, Sharma et al ${ }^{[10]}$, Nagaria et al ${ }^{[11]}$ Sample size of Madhu et $\mathrm{al}^{[12]}$, Dahal et $\mathrm{al}^{[17])}$ were small, but Drotavarine had shorter drug delivery interval than other group.

Table 10 Mode of Delivery

\begin{tabular}{|l|c|c|c|c|c|c|}
\hline \multirow{2}{*}{ STUDY } & \multicolumn{3}{|c|}{ GROUP A } & \multicolumn{3}{c|}{ GROUP B } \\
\cline { 2 - 7 } & NVD & LSCS & INST & NVD & LSCS & INST \\
\hline $\begin{array}{l}\text { Dahal et al (2013) } \\
{[17]}\end{array}$ & $100 \%$ & - & - & $96 \%$ & $2 \%$ & $2 \%$ \\
\hline $\begin{array}{l}\text { Kalhon et al } \\
(2015)^{[19]}\end{array}$ & $96 \%$ & - & $4 \%$ & $98 \%$ & - & $4 \%$ \\
\hline Patil et al (2015) $)^{[16]}$ & $96 \%$ & - & $4 \%$ & $92 \%$ & - & $8 \%$ \\
\hline $\begin{array}{l}\text { Selvaraj et al (2016) } \\
{[18]}\end{array}$ & $94 \%$ & - & $6 \%$ & & & \\
\hline $\begin{array}{l}\text { Present study } \\
(2016)\end{array}$ & $98 \%$ & $2 \%$ & - & $98 \%$ & $2 \%$ & - \\
\hline
\end{tabular}

Present study, Group A 98\% delivered vaginally, $2 \%$ LSCS. In group B 98\% delivered normally and $2 \%$ LSCS. results comparable to those of other authors. ${ }^{[17,19,16,18]}$

Table 11 Third Stage Complications

\begin{tabular}{|l|c|c|c|c|}
\hline Study & GROUP & PPH & $\begin{array}{c}\text { Cervical } \\
\text { Tear }\end{array}$ & $\begin{array}{c}\text { Retained } \\
\text { Placenta }\end{array}$ \\
\hline $\begin{array}{l}\text { Sharma et al (2001) } \\
{[10]}\end{array}$ & $\mathrm{A}$ & $18 \%$ & - & - \\
\cline { 2 - 5 } & $\mathrm{B}$ & - & - & - \\
\hline $\begin{array}{l}\text { Jayshree et al (2013) } \\
[20]]\end{array}$ & $\mathrm{A}$ & $3.03 \%$ & $2.08 \%$ & - \\
\cline { 2 - 5 } & $\mathrm{B}$ & - & $2.29 \%$ & - \\
\hline \multirow{2}{*}{ Dahal et al (2013) [17] } & $\mathrm{A}$ & $2.3 \%$ & - & - \\
\cline { 2 - 5 } & $\mathrm{B}$ & $2.5 \%$ & - & - \\
\hline \multirow{2}{*}{ Naga et al (2014) [13] } & $\mathrm{A}$ & - & $6.66 \%$ & - \\
\cline { 2 - 5 } & $\mathrm{B}$ & - & - & - \\
\hline \multirow{2}{*}{ Present study (2016) } & $\mathrm{A}$ & - & - & $1.02 \%$ \\
\cline { 2 - 5 } & $\mathrm{B}$ & - & - & - \\
\hline
\end{tabular}

No PPH/ cervical tear in either group in our study. $1.02 \%$ had retained placenta in Group A.

Authors ${ }^{[20,17,13]}$ showed incidental PPH, cervical tear, retained placenta not attributed to drugs. Sharma et $\mathrm{al}^{[10]}$ noticed $\mathrm{PPH}$ in $18 \%$ of Drotavarine patients but no causal relationships ascertained..

Table 12 Maternal Side Effects

\begin{tabular}{|c|c|c|c|c|c|c|c|}
\hline Study & Group & 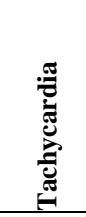 & 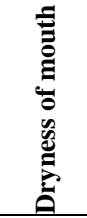 & 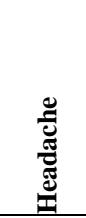 & 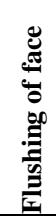 & 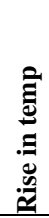 & 泀 \\
\hline \multirow{2}{*}{$\begin{array}{l}\text { Sharma et al } \\
(2001)^{[10]}\end{array}$} & A & $4 \%$ & - & $4 \%$ & - & - & - \\
\hline & $\mathrm{B}$ & $20 \%$ & $10 \%$ & - & $4 \%$ & - & - \\
\hline \multirow{2}{*}{$\begin{array}{l}\text { Nagaria et al } \\
(2009)^{[11]}\end{array}$} & $\mathrm{A}$ & $3 \%$ & $4 \%$ & - & - & - & $4 \%$ \\
\hline & $\mathrm{B}$ & $22 \%$ & $22 \%$ & - & $2 \%$ & - & $7 \%$ \\
\hline \multirow{2}{*}{$\begin{array}{l}\text { Dahal et al } \\
(2013)^{[17]}\end{array}$} & $\mathrm{A}$ & $4 \%$ & - & - & - & - & - \\
\hline & $\mathrm{B}$ & $21 \%$ & - & - & - & $5 \%$ & - \\
\hline \multirow{2}{*}{$\begin{array}{l}\text { Patil et al } \\
(2015)^{[16]}\end{array}$} & $\mathrm{A}$ & $12 \%$ & - & $6 \%$ & - & - & - \\
\hline & $\mathrm{B}$ & $28 \%$ & $12 \%$ & $8 \%$ & - & - & - \\
\hline \multirow{2}{*}{$\begin{array}{l}\text { Present study } \\
(2016)\end{array}$} & $\mathrm{A}$ & $5.10 \%$ & $6.12 \%$ & $4.08 \%$ & - & - & - \\
\hline & B & $8.16 \%$ & $3.06 \%$ & $1.02 \%$ & - & - & - \\
\hline
\end{tabular}

Our study: Group B drugs caused more tachycardia than Drotavarine, also observed by

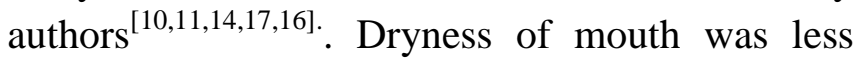
with combination drugs than other studies ${ }^{[10,11,16]}$ More women had headache(Drotavarine Group) also observed by authors. ${ }^{[10,16]}$

\section{Neonatal Outcome}

Present study: No adverse APGAR score in either group. 1 NICU admission due to LBW (Group 
B),. Naga et $\mathrm{al}^{[13])}$, Kaur et $\mathrm{al}^{[14]}$ found no adverse APGAR score or NICU admission. So drugs were found safe..

\section{Summary and Conclusion}

Study was conducted to compare the effect of Drotavarine hydrochloride and combination of Hyoscine-N-butyl bromide with Valethamate bromide on active phase and find maternal and fetal side effects.

200 women divided into two groups of 100 each. Group A received Drotavarine hydrochloride $40 \mathrm{mgI} / \mathrm{M}, 2$ doses 2 hours apart ( $2^{\text {nd }}$ if required). Group B given combination of Hyoscine-N-butyl bromide $40 \mathrm{mg}$ and Valethamate bromide $16 \mathrm{mg}$ $\mathrm{i} / \mathrm{m}, 2$ doses 2 hours apart(2nd if required).

1) Majority were between 20-25 years of age ( both groups). Mean age was 24.38 \pm 3.12 years in Group A and 23.25 \pm 2.88 in B.

2) $45 \%$ were primigravidae (Group A), $51 \%$ in Group B. Multigravidae 55\% in Group A and $49 \%$ in Group B.

3) Mean POG was $38.56 \pm 1.03$ and $38.69 \pm 0.90$ weeks in Group A and B respectively.

4) At recruitment, mean cervical effacement was $\quad 61.20 \pm 10.37 \% \quad$ (Group A), $60.20 \pm 10.04 \%$ (Group B) which was comparable.

5) $98 \%$ delivered vaginally in both groups. $2 \%$ LSCS (each group) and excluded No instrumental deliveries.

6) Mean dose No;. $1.36 \pm 0.48$ (Group A), $1.83 \pm 0.37 \quad$ (Group B), statistically significant.

7) Mean rate of dilatation was more in Group A than Group B which was significant.

8) Mean Active phase was significantly shorter (112.70 \pm 53.09 mins) in Group A than Group B. (171.44 \pm 57.24$)$.

9) Drug-delivery interval was shorter in Group A than Group B. Statistically significant.
10) No significant difference in second and third stages between the two groups.

11) No PPH or cervical tear.

12) No serious maternal /fetal side effects in either group.

\section{Conclusion}

Drotavarine hydrochloride and combination of Hyoscine-n-Butyl bromide with Valethamate bromide can be used to shorten the duration of active phase of labor in both primigravidae and multigravidae. Drotavarine found to be better drug, hence, can be used to reduce agony of laboring woman without any significant side effects on the mother or the fetus'.

\section{Grants: Nil}

\section{References}

1. Shahul R, Chapman A. Role of drugs in acceleration of normal labor. J Obstet Gynecol Ind 1984; 34:501-504.

2. Philpot RH, Castle WM. Cervicographs in the management of labor in primigravida; the alert line for detecting abnormal labor. J Obstet Gynecol Brit C'wlth 1972; 79:592.

3. World Health Organization. Managing Prolonged and Obstructed Labor. Education material for teachers of midwifery: midwifery education modules. - 2nd ed,2008:54-64

4. Mitchell MD, Flint, APF, Bibby J et al. Rapid increase in plasma PG concentration after vaginal examination and amniotomy. Br Med J 1997; 2:1183-84.

5. Busowskie JD, Passons NJ. Amniotomy to induce labor. Clin Obstet Gynecol 1995; 38(2):256-49.

6. Fraser WD, Sauve R, Singh PW et al. A randomized controlled trial of early anatomy. $\mathrm{Br} \mathrm{J}$ Obstet Gynecol 1991; 98(1):84-91. 
7. Shykin JM, Petrie RH. Oxytocin to induce labor. Clin Obstet Gynecol 1995; 38(2):202-12.

8. Cheng YW, Shaffer BL, Bryant AS, Caughey AB. Length of the first stage of labor and associated prenatal outcomes in nulliparous women. Obstet and Gynecol 2010; 116:1127-35.

9. Vellanki VS. Comparison of buscopan and epidosin on cervical dilatation in labour. Global journal of medical research 2010;10(2): 18-21.

10. Sharma JB, Pundir P, Kumar A, Murthy NS. Drotavarine hydrochloride v/s Valethamate bromide in acceleration of labour. Int J Gynae Obst 2001 Sep; 74(3):255-60.

11. Nagaria T, Jaiswal J. To compare and evaluate the efficacy and safety of Drotavarine and Valethamate bromide. J Obst Gynae India 2009; 59(4):324-33.

12. Madhu C, Mahavarkar S, Bhave S. A randomized controlled study comparing Drotavarine hydrochloride and Valethamate bromide in the augmentation of labor. Arch Gynecol Obstet.2010;282 (1):11-5

13. Naga P, Shivanna BS, Subbappa K, Gopal N. Comparative Study of Efficacy of Combination of Valethamate Bromide \& Buscopan with Drotavarine Hydrochloride on Cervical Dilatation in Active Labor. IOSR-JDMS 2014 Oct; 13(10):27-29.

14. Kaur S, Bajwa SK, Kaur P, Bhupal S. To Compare the Effect of Camylofin Dihydrochloride (Anafortan) with Combination of Valethamate Bromide (Epidosin) and Hyoscine Butyl-N-Bromide (Buscopan) on Cervical Dilation. J Clin Diagn Res. 2013 Sep; 7(9): 1897-1899.

15. Edessy $M$ et al. Different modalities in first stage enhancement of labor. Gen. Health Med Sci.,2015;(2):1-4
16. Patil AB, Kalhon P, Patel R. Comparative study of Drotavarine Hydrochloride and Valethamate Bromide in progress of labor. International Journal of Recent Trends in Science and Technology, 2015;14(3):678682

17. Dahal $P$ et al. Health Renaissance Efficacy of Drotavarine hydrochloride \& Valethamate bromide in labor. 2013; 11(1); 38-42.

18. Selvaraj SM, Natarajan S. Comparison of Drotavarine hydrochloride and Valethamate bromide on cervical dilatation. Journal of Evolution of Medical and Dental Sciences, 2016;5(52):3391-3394

19. Kalhon P ,Patil AB, Patel R. Efficavy of intramuscular Drotavarine use for cervical dilatation in first stage of labor. International Journal of Recent Trends in Science and Technology, 2015;14(3): 670674.

20. Jayashree S, Ajjammanavar V, Sujatha MS. Int J Biol Med Res 2013; 4(2):32153218 